\title{
Correction to: A prospective randomised control trial to compare the perioperative outcomes and ergonomic challenges between triangular versus midline port placement in total extra- peritoneal repair of uncomplicated inguinal hernia
}

\author{
Sapna Singh ${ }^{1} \cdot$ Akshay Anand $^{1}$ • Awanish Kumar ${ }^{1} \cdot$ Ajay K. Pal ${ }^{1} \cdot$ Manish K. Agrawal $^{1} \cdot$ Sanjeev Kumar $^{1}$. \\ Harvinder S. Pahwa ${ }^{1} \cdot$ Abhinav A. Sonkar ${ }^{1}$
}

Published online: 11 June 2020

(c) Springer Science+Business Media, LLC, part of Springer Nature 2020

\section{Correction to: Surgical Endoscopy \\ https://doi.org/10.1007/s00464-020-07525-4}

The title "A prospective randomised control trial to compare the perioperative outcomes and ergonomic challenges between triangular versus midline port placement in total extra-peritoneal repair of uncomplicated unilateral inguinal hernia" should have read "A prospective randomised control trial to compare the perioperative outcomes and ergonomic challenges between triangular versus midline port placement in total extra-peritoneal repair of uncomplicated inguinal hernia."

It is correct here.

Publisher's Note Springer Nature remains neutral with regard to jurisdictional claims in published maps and institutional affiliations.

Sapna Singh and Akshay Anand are co-first authors.

The original article can be found online at https://doi.org/10.1007/ s00464-020-07525-4.

Awanish Kumar

awanishkr79@gmail.com

1 Department of General Surgery, King George's Medical

University, Lucknow, UP 226003, India 